East African Medical Journal Vol 77 No. 1 January 2000

PREDICTIVE INDICES IN TRAUMATIC INTRACRANIAL HAEMATOMAS

G. O. Igun, FWACS, Department of Surgery, The Neurosurgical Unit, Jos University Teaching Hospital, P. M. B. 2076 Jos. Nigeria.

\title{
PREDICTIVE INDICES IN TRAUMATIC INTRACRANIAL HAEMATOMAS
}

\author{
G. O. IGUN
}

\begin{abstract}
Objective: To identify factors of predictive value in the early diagnosis of traumatic intra-cranial haematomas (ICH).

Design: A retrospective study of patients with positive exploratory burr hole findings of ICH.

Setting: Jos University Teaching Hospital, Nigeria between January 1988 and December 1998.

Patients: Two hundred and six patients.

Main outcome measures: The demographic data of patients, duration of presentation, Glasgow Coma Scale (GCS), causes, clinical features and mortality characteristics of patients were analysed.

Results: There was a bi-modal age distribution with a mean of 26 years. Male: female ratio was 2:1. Vehicular accidents accounted for $72 \%$ of $\mathrm{ICH}$ and falls for $22 \%$. Sixty seven per cent recorded a GCS of 14-15 out of a total of 175 GCS recorded. A brief history of loss of consciousness was obtained in $33 \%$, headaches $49 \%$, deterioration in conscious level $91 \%$, seizures $53 \%$, cranial nerve deficits $11 \%$ and hemiplegia in $22 \%$. Significant lateralising signs occurred within 72 hours. Extra-dural haematomas constituted $37 \%$ and were associated with cranial vault fractures in all cases. Subdural haematomas constituted $60 \%$, with skull fractures in eight per cent and intracerebral haematomas occurred in three per cent. A mortality of seven per cent was recorded for all patients with ICH.

Conclusion: Traumatic ICH is associated with raised intracranial pressure in many patients.
\end{abstract}

\section{INTRODUCTION}

Patients who develop intracranial haematomas (ICH) sequel to head injuries are perpetually threatened by the development of mid-brain cones if left untreated. The resultant fatality that might attend this complication of head injury might be described as unfortunate since the resultant effect of secondary brain damage is potentially reversible with treatment(1). It is therefore logical to assert that the prevention and treatment of intracranial haematomas is the key to reduction of the mortality from head injury.

This gloomy picture of the outcome of $\mathrm{ICH}$ is further compounded by the fact that the neurosurgical centres in Nigeria are few and far between, each one covering a large area and population. Few, if any of these centres were in possession of modern imaging techniques such as computed tomograph (CT) scan(2) until quite recently. Given these existing state of affairs, the recognition of those patients with head injury liable to develop ICH should be the essential first step in reducing mortality figures, especially as it concerns referring hospitals. The objective of this study was to identify factors of predictive value in the early diagnosis of ICH in head injured patients. A greater awareness of these predictive indices in $\mathrm{ICH}$ will speed up referral of such helpless patients to tertiary centres where definitive treatment could be obtained. Such indices will also help to streamline the indications for use of CT scan in many areas of the tropics where the cost of this diagnostic aid is at present too high for routine use in all head injured patients.

\section{MATERIALS AND METHODS}

A retrospective study of 206 patients with ICH admitted or referred to the Jos University Teaching Hospital (JUTH) was undertaken. Cases accrued to the study between January 1, 1988 and December 23, 1998. Diagnosis of ICH was based on operative findings following burr hole exploration, which was performed on a total of 268 patients with suspected traumatic ICH. The Glasgow Coma Scale (GCS) was used in assessing the conscious level of patients and in following serial trends in brain function of the patients studied. In children under four years of age, the Advanced Trauma Life Support Modification of the GCS was employed(3). Plain xrays of the skull was undertaken in all patients following full resuscitation. The indication for burr hole exploration in these patients were: neurological deficits on presentation with or without evidence of alteration of consciousness and deterioration in conscious level on admission. Although realising the inherent errors in interpretation of retrospective data, an attempt was nevertheless made to correlate the age, sex, causes, duration of head injury before presentation, 
Glasgow Coma Scale, and neurological abnormality in patients, with a view to determining predictive indices for the development of ICP in these patients.

\section{RESULTS}

The age distribution of patients with $\mathrm{ICH}$ is illustrated in Figure 1. There was a bi-modal distribution with $23 \%$ of patients in the (1-10) years age bracket and $24 \%$ in the bracket of (21-30) years. The mean age of patients on presentation was 26 years with a range of eight months to 75 years. There were 137 males and 69 females constituting a M:F ratio of 2:1. One hundred and seventy one of 206 (83\%) patients were brought directly from the scene of the accident while $35(17 \%)$ were referred. Table 1 indicates that the duration between the event and presentation to hospital was less than 12 hours in patients admitted directly, more than 12 hours in 28 (14\%) patients referred and indeterminate in seven $(3 \%)$ patients.

Figure 1

Age distribution of patients with ICH at JUTH, 1988-1998

Table 1

Duration before presentation in patients with ICH: JUTH: 1988-98

\begin{tabular}{lr}
\hline Duration (hrs) & No. \% \\
\hline $1-6$ & $122(59)$ \\
$7-12$ & $49(24)$ \\
$13-18$ & $10(5)$ \\
$19-24$ & $10(5)$ \\
$25-30$ & $8(4)$ \\
Indeterminate & $7(3)$ \\
\hline Total & $206(100)$ \\
\hline
\end{tabular}

Percentage in brackets

Table 2 shows the causes of head injury in patients with ICH. Vehicular accidents accounted for a total of 155 of 206 (75\%), patients 135 of whom were occupants, falls occurred in $22 \%$ and assault in three per cent of all cases of ICH.
Table 2

Causes of head injury in patients with ICH: JUTH: 1988-98

\begin{tabular}{lr}
\hline Cause & Number $(\mathrm{N}=206)$ \\
\hline Vehicular $(\mathrm{n}=155)$ & \\
Occupants & $135(65)$ \\
Pedestrians & $8(4)$ \\
Motor cycle & $12(6)$ \\
Non vehicular $(\mathrm{N}=51)$ & $45(22)$ \\
Falls & $6(3)$ \\
Assault & $206(100)$ \\
\hline Total
\end{tabular}

The neurologic findings on admission in patients with ICH are indicated in Table 3, which indicates distribution of patients according to the Glasgow Coma Score (GCS) and positive neurologic findings recorded. The GCS was documented in 175 patients and categorisation of the severity of the head injury into the three types was achieved as follows: GCS (14-15) minor; (9-13) moderate and severe(3-8). We have subdivided the GCS of severe head injury into (35) and (68) in order to further relate this category of head injury to prognosis. Most of the patients with $\mathrm{ICH}$ studied had minor head injury $(67 \%)$ followed by the

Table 3

GCS and neurologic findings in patients with ICH; JUTH: 1988-98

\begin{tabular}{lr}
\hline GCS & $(\mathrm{n}=175)$ \\
\hline $3-5$ & $3(2)$ \\
$6-8$ & $5(3)$ \\
$9-13$ & $50(28)$ \\
$14-15$ & $117(67)$ \\
Neural abnormalities: & $\mathrm{N}=206)$ \\
History of loss of consciousness & $67(33)$ \\
Headache & $101(49)$ \\
Behavioural abnormality & $5(2)$ \\
Decreased consciousness & $187(91)$ \\
Seizures & $26(53)$ \\
Aphasia & $14(7)$ \\
Cranial nerve deficits & $16(8)$ \\
III N & $6(3)$ \\
VII N & 46 \\
Hemiplegia & $4(2)$ \\
Urinary incontinence & $2(2)$ \\
Cerebellar ataxia & $21(10)$ \\
Papilloedema & $19(9)$ \\
None &
\end{tabular}

Some patients had more than one symptom

moderate form in $28 \%$. There was a history of brief loss of consciousness (less than five minutes) in $33 \%$ of patients. The headache that occurred in a total of $101(49 \%)$ constituted the predominant complaint in 117 patients $(86 \%)$ that were conscious. It was persistent, protracted and severe (disturbing sleep) but was of no localising value in all cases. Behavioural abnormalities occurred in two per cent of patients. Deterioration in conscious level by a number of 2 or more in the GCS 
occurred in $91 \%$ of patients. Seizures occurred in $53 \%$ of patient and whether they were generalised or focal was of no localising value in patients thus affected. Aphasia occurred in seven per cent and was of localising value in 10 patients. Cranial nerve deficits occurred in a total of $22(11 \%)$ patients; in 16 patients third nerve deficits were recorded and the remaining six patients sustained seventh nerve deficits of the upper motor-neurone type. Hemiplegia was recorded in $22 \%$, urinary incontinence in two per cent and cerebellar ataxia in one per cent. Papilloedema was recorded in $10 \%$. In $19(9 \%)$ of 206 patients, no neural deficits were recorded apart from deterioration in the level of consciousness.

The incidence and type of fractures in patients with $\mathrm{ICH}$ is indicated in Table 4. Eighty seven patients (40\%) sustained skull fractures, the distribution of which are shown. Linear fractures of the squamous temporal bone occurred in $72(34 \%)$ patients with intracranial haematomas. Sixty six fractures where associated with extra-dural haematomas (EDH) and six with subdural haematomas (SDH). Sagittal fractures of the cranial vault occurred in three (1\%) patients; two were associated with EDH and one with a SDH. Nine stellate fractures of the vault (four per cent) all resulted in nine cases of EDH. Three depressed fractures of the cranial vault (one per cent) were associated with three cases of contra-coup subdural haematomas. Overall, 87 fractures of different types were associated with 77 cases of EDH and 10 cases of SDH.

\section{Table 4}

Incidence of fracture types in ICH; JUTH: 1988-98

\begin{tabular}{lccc}
\hline Fracture & EDH $(\mathrm{n}=77)$ & SDH $(123)$ & Total $(\mathrm{n}=87)$ \\
\hline Linear & & & \\
Squamous & 66 & 6 & $72(34$ \\
Sagittal & 2 & 1 & $3(1)$ \\
Stellate & 9 & 0 & $9(4)$ \\
Depressed & 0 & 3 & $3(1)$ \\
\hline Total & $77(100)$ & $10(8)$ & $87(40)$ \\
\hline
\end{tabular}

Table 5

Exploratory burr hole findings and outcome in patients with suspected ICH: JUTH 1988-1998

\begin{tabular}{lr}
\hline Finding & No. $(\mathrm{n}=268)$ \\
\hline Negative & $62(23)$ \\
Brain swelling & 47 \\
Contusion & $15(25)$ \\
Mortality & $3(1)$ \\
Positive & 206 \\
Extradural haematoma & $77(37)$ \\
Subdural haematoma & $123(60)$ \\
Intracerebral haematoma & $6(3)$ \\
Mortality & $15(7)$ \\
\hline
\end{tabular}

*Five patients sustained chronic SDH
Table 5 summarises the exploratory burr hole findings in the 268 patients with suspected $\mathrm{ICH}$. Negative exploratory burr hole was recorded in 62 $(23 \%)$ patients with a hospital mortality in three $(1 \%)$ patients. Hemiplegia which occurred in 10 patients (16\% and aphasia in three patients (1\%) were recorded on presentation in the 13 patients with contusions of the brain. In the 206 patients (77\%) with ICH reported in this study, EDH was recorded in 77 (37\%) patients, SDH in 123 patients (60 per cent) including five cases of chronic SDH and intracerebral haematomas of the superficial type in six patients $(3 \%)$.

Table 6

Mortality characteristics of patients with ICH: JUTH; 1988-98

\begin{tabular}{lr}
\hline Characteristic & $(\mathrm{n}=15)$ \\
\hline Duration of presentation & \\
(>24 hours) & $8(53)$ \\
GCS (3-5) & $3(20)$ \\
$(6-8)$ & $4(26)$ \\
Increased ICP & $12(80)$ \\
SDH & $11(73)$ \\
EDH & $4(26)$ \\
Clot (> 150 mls) & $4(20)$ \\
Multiple injuries & $3(20)$ \\
\hline
\end{tabular}

ICP = Intracranial pressure

Hemiplegia which was recorded in $36(17 \%)$ patients and aphasia in $11(5 \%)$ patients tended to develop post admission within 72 hours in the 47 patients with ICH. A hospital mortality of $15(7 \%)$ patients of the total number of 206 patients was recorded. Eight of these presented after 24 hours of injury. Three patients had a GCS of(3-5) and four with a GCS of(6-8). Evidence of raised intrapial pressure was recorded in 12 patients a "tight brain" at exploration; eleven of the cases were $\mathrm{SDH}$ and four were EDH. In four cases the amount of clot evacuated from the brain was more than $150 \mathrm{mls}$ and three fatalities were associated with multiple injuries (Table 6).

\section{DISCUSSION}

The epidemiological indices of ICH as gleaned from this study should be noted. Intracranial haematoma was found to have a bimodal age distribution with about $23 \%$ of patients in the age group 1-10 years and 2130 years age respectively. Our findings in the younger age group do not agree with that of other series that there is a low incidence of mass lesion in children(4). The mean age of presentation of patients with $\mathrm{ICH}$ was 26 years and the sex ratio $\mathrm{M}: \mathrm{F}$ was $2: 1$ reflecting the category of patients thus afflicted. Patients with ICH in this study were those who sustained head injuries from vehicular accident $(75 \%)$, falls especially in children (22\%) and assaults (3\%). Vehicular accidents and falls on the head represent severe injuring forces in which the brunt of injury is taken by the skull bone leading to injury to the middle meningeal artery or sagittal sinus, with resultant EDHS(1). Assaults on the 
other hand present mild injuring forces which are not enough to fracture the skull but could lead to sudden displacement and distortion of the brain, resulting in direct coup and injuries or cerebral venous injuries with resultant SDH.

Sixty seven per cent of the patients in this study presented with a GCS of 14-15, followed by another $28 \%$ with a GCS of $9-13$. These findings agree with what is widely known about acute EDH and $\mathrm{SDH}$ (occurring within 48 hours). Patients are usually clear in mind at the outset only to lapse into unconsciousness when the mass effect of ICH develops. The satellite clinical features of these patients which we have found useful included headache, which occurred in $86 \%$ of all patients that were conscious on admission(5). Deterioration in the level of consciousness occurred in $91 \%$ and seizures were recorded in $53 \%$. We have noted a syndrome of behavioural abnormalities, urinary incontinence and cerebellar ataxia to be diagnostic and of specific localising value in ICH that are situated in the region of the pre-motor cortex in about two per cent of patients. The diagnosis of ICH must be based on a high index of suspicion, especially when one or more of the clinical features mentioned exist. Other features that might be of value late in the course of development of ICH include cranial nerve deficits (III and VII) in $11 \%$, aphasia in seven per cent and hemiplegia in $22 \%$. Cranial nerve deficits, aphasia and hemiplegia which tended to develop over a period of 72 hours post-head injury tended to be more diagnostic of ICH and were of great localising value. Patients who on the other hand presented with early neural deficits on admission in the face of a stable GCS nearly always had sustained cerebral contusions as observed from our negative exploratory burr hole findings. Papilloedema, which occurred in $10 \%$ is a late sign that nearly always signalled impending doom in patients with $\mathrm{ICH}$ with mid-brain cloning.

Extra-dural haematomas were associated with fractures in all the patients. The fractures generally conformed to the situation of vascular structures that had been damaged as a result of the impact on the skull by injuring forces. Linear fractures of the squamous temporal bone with damage to the middle meningeal artery and sagittal fractures with damage to the superior sagittal sinus were encountered in this study as examples. We however agree that EDH can occur without evidence of skull fractures as has been observed in other studies(6).

We recorded a $23 \%$ negative burr hole finding in this study. This figure can be further decreased if attention is paid to some of the clinical indices in this study that might predict ICH. In particular, we emphasise the fact that neurological deficits in patients with $\mathrm{ICH}$ take about 72 hours from the time of injury to develop and are more relevant in the face of concomitant decrease in the level of conscious. When doubt exists however exploratory burr hole should be undertaken because the mortality in this series of one per cent is acceptable and it affords an opportunity to manage brain swelling by performing sub-temporal decompression at the same operation. Brain contusions recorded in absence of clots could account for neural deficits which were present on admission together with a stable GCS.

For positive burr hole exploration, EDH constituted $37 \%$ and SDH 60 per cent; the ratio of SDH to EDH was 2:1 Mendeow et al (1) in the UK and Mustaffah et al(6) in Ghana reported ratios as high as 3:1 and $6: 1$, respectively. We recorded only five patients with chronic SDH. This is at variance with the affirmation by Adeloye et al(5) that in West Africa, chronic SDH is the commonest cause of ICH. Superficial intracerebral haematoma was recorded in three per cent of patients. Hospital morality in patients with $\mathrm{ICH}$ was seven per cent and was found to be related to factors like a long duration of presentation (after 24 hours), low GCS(3-8), features of increases ICP, the evacuation of large collections of clot (>150 mls) and associated presence of multiple injuries. These extracranial injuries have an additive effect in worsening the outcome from head injuries(8).

In the final appraisal of the results of this study, it must be recalled that ICH is associated with increased intra-cranial pressure (ICP) in many patients with head injuries(9). The natural history of ICP following head injury may be towards spontaneous reduction or a progressively down hill deterioration with ischaemic brain damage when the autoregulatory mechanisms of the brain fail(1). A localised intra-cranial lesion might also produce brain shifts with subfalcine, transtentorial or tonsilar herniation leading to medullary compression with respiratory arrest and death from ICH. All these call for a high index of suspicion on the part of the attending surgeon who must now no longer wait for the effects of secondary brain damage but recognise the circumstances which are likely to lead to it and forestall them. Computed tomography scan is at present the most valuable diagnostic aid to employ in localizsation of ICH but when not available, clinical predictive indices should be employed(7).

\section{REFERENCES}

1. Mendelows A.D. and Teasdale G.M. Pathophysiology of head injuries. Brit. J. Surg. 1983; 641-650.

2. Teasdale G.M. Murray G. and Anderson E. et. al. Risks of acute traumatic intracranial haematoma in children and adults; implications for managing head injuries.

3. American College of Surgeons Advanced Trauma Life support student manual 5th end. American College of Surgeons; Chicago: Illinois, 1993.

4. Luerssen J.G. Klauber M.R. and Marshall L. Outcome from head injury related to patients age. A longitudinal prospective study of adult and paediatric head injury. J. Neulosurg. 1986; 68:409-16.

5. Adeloye, A. Complication of head injury. In: Neurosurgery in Africa 1989. University Press Ibadan, 32-68.

6. Mustaffah, J.F.O. Surgery of subdural and epidural haematoma. Med. d'Afri Noire; 1988: 23:203-204.

7. Christopher L.G. and Cummins B. Initial assessment and management of the severely head injured patient. Brit. J. Hosp. Med. 1995; 53:102-8. 